TITLE:

\title{
Wide-range frequency selectivity in an acoustic sensor fabricated using a microbeam array with non- uniform thickness
}

\section{$\operatorname{AUTHOR}(S)$ :}

Shintaku, Hirofumi; Kobayashi, Takayuki; Zusho, Kazuki; Kotera, Hidetoshi; Kawano, Satoyuki

\section{CITATION:}

Shintaku, Hirofumi ... [et al]. Wide-range frequency selectivity in an acoustic sensor fabricated using a microbeam array with non-uniform thickness. Journal of Micromechanics and Microengineering 2013, 23(11): 115014.

\section{ISSUE DATE:}

2013-11-01

URL:

http://hdl.handle.net/2433/192999

\section{RIGHT:}

(c) 2013 IOP Publishing Ltd.; この論文は出版社版でありません。引用の 際には出版社版をご確認ご利用ください。; This is not the published version. Please cite only the published version. 


\section{Wide-range frequency selectivity in acoustic sensor fabricated using microbeam array with non-uniform thickness}

Hirofumi Shintaku ${ }^{1 *}$,Takayuki Kobayashi ${ }^{2}$, Kazuki Zusho ${ }^{2}$, Hidetoshi Kotera ${ }^{1}$, and Satoyuki Kawano $^{2}$

${ }^{1}$ Graduate School of Engineering, Kyoto University, Nishikyo-ku Kyoto University Katsura, Kyoto, 615-8540 Japan, shintaku@me.kyoto-u.ac.jp

${ }^{2}$ Graduate School of Engineering Science, Osaka University, 1-3 Machikaneyama, Toyonaka, Osaka, 560-8531 Japan, kawano@me.es.osaka-u.ac.jp

${ }^{*}$ Corresponding Author: Hirofumi Shintaku

\section{Abstract}

In this study, we have demonstrated the fabrication of a microbeam array (MBA) with various thicknesses, and investigated it suitability for an acoustic sensor with wide-range frequency selectivity. For this, an MBA composed of 64 beams, with thickness varying from 2.99-142 $\mu \mathrm{m}$, was fabricated by using single gray-scale lithography and thick negative photoresist. The vibration of beams in air was measured by using a laser Doppler vibrometer, and the resonant frequencies of beams were measured to be from $11.5 \mathrm{kHz}$ to $290 \mathrm{kHz}$. Lastly, the frequency range of the MBA with non-uniform thickness was 10.9 times that of the MBA with uniform thickness.

Keywords: acoustic sensor, vibration, resonance, gray-scale lithography, 3D fabrication 


\section{Introduction}

The basilar membrane, which is a flexible membrane in the cochlea of the inner ear, plays a key role in sensing the frequency of the sound. The sensitivity of the basilar membrane is determined by the location and range of the local characteristic frequency (CF) at which the ear is most sensitive to sound vibrations. For human beings, the CF ranges from $20 \mathrm{~Hz}$ to $20 \mathrm{kHz}$. The basilar membrane assumes a long and trapezoidal shape, if extended along its length. Such changes in its conformation is responsible for the variations in CF. In addition, thickness and mechanical property of the basilar membrane vary along the length of the duct, resulting in the relatively wide frequency range of the auditory system. This features the cochlea as a remarkable acoustic transducer with a compact and robust design. Inspired by the cochlear functions of the basilar membrane, several researchers have attempted to mimic the mechanical cochlear system to realize a cochlea-like frequency analyzer ${ }^{1-7}$. Technically, the frequency analysis in such devices is accomplished using a membrane ${ }^{2,7}$, a beam array ${ }^{3,6}$ and a membrane with beams ${ }^{1,4,5}$, which vibrates by applying sound.

Recently, researchers have developed a bionic auditory membrane (BAM), which could be used as a sound processor for novel implantable artificial cochlea ${ }^{2,8,9}$. The BAM consists of a flexible membrane made of a piezoelectric material and an electrode array. BAM can generate an electrical signal by applying sound, making it viable to replace the functions of hair cells, which are sensory cells in the cochlea ${ }^{10}$. At the same time, the BAM realizes the frequency selectivity by the trapezoidal geometry, which is designed by mimicking the shape of a basilar membrane. In our previous study reported elsewhere ${ }^{2}$, we had confirmed the frequency selectivity of BAM. However, it was observed that the BAM functioned in a very narrow frequency range of $6.6 \mathrm{kHz}$ to $19.8 \mathrm{kHz}$, compared with the human audible frequency. One of the main reasons for this narrow frequency range was the uniform thickness of the BAM. This did not mimic the basilar membrane, in which the 
thickness changes with frequency and location. To integrate acoustic sensors with wide-range frequency selectivity in a small space, the thickness should change by location.

Herein, we have demonstrated the fabrication of a microbeam array (MBA) with various thicknesses using a simple method that employs gray-scale lithography with a negative photoresist. We reported brief, preliminary results in conference proceedings ${ }^{11,12}$, and here present detailed study of the fabrication and device characteristics. So far, several approaches have been proposed for the microfabrication of three-dimensional structures. They include: 1) multi-layer/multi-exposure process $\left.{ }^{13-16}, 2\right)$ inclined/rotated UV lithography ${ }^{17}$, 3) gray-scale lithography ${ }^{18-20}$, and 4) moving mask lithography ${ }^{21}$. Among these methods, we have adopted gray-scale lithography, since it is straightforward to implement into a standard photolithography process. In this study, we could observe that the range of the resonant frequency greatly expands with changes in the thickness of the beam array. To the best of our knowledge, the MBA proposed in this study is the first demonstration of a life-sized physical model of a basilar membrane with changing thickness.

\section{Experimental Methods}

The conceptual illustration of the different stages involved in the fabrication of flexible beams is shown in Figs. 1(a) and (b). The grayscale photomask has a transmittance schematically depicted in the top of Fig. 1(a). In this study, we used a negative photoresist that becomes insoluble in a development solution by UV-exposure and thermal treatment. Generally, negative photoresists have low absorption coefficients for UV light, such as i-line of $365 \mathrm{~nm}$ and g-line of $436 \mathrm{~nm}$, making them unsuitable for gray-scale UV lithography. In order to exploit UV light with shorter wavelengths $(<350 \mathrm{~nm})$, which have relatively high absorption coefficients, we used a band pass filter (330WB80, OMEGA Optical) with the maximum transmittance at $330 \mathrm{~nm}$ and a bandwidth of $80 \mathrm{~nm}$. By modifying the UV light, exposed energy in the photoresist was gradually decreased from the surface toward the 
substrate. Consequently, the negative photoresist under the gray-scale pattern was fully exposed only near the surface, leaving it under exposed near the substrate. Since the part of fully exposed photoresist turns to be insoluble and that under exposure can still dissolve in the development solution, the fully exposed part near the surface construct a solid structure and is released from the substrate after the development. The thickness of the solid structure is controlled by transmittance of the gray-scale patterns, where lower transmittance results in thicker structures. While the BAM demonstrated in our previous study had a membrane-like structure, the device fabricated in this study is designed as an array of microbeams, to facilitate the development process in which the photoresist under the beam dissolves from the open ends. Furthermore, the beam structure is useful to evaluate the frequency characteristics by comparing theoretical and experimental results. 

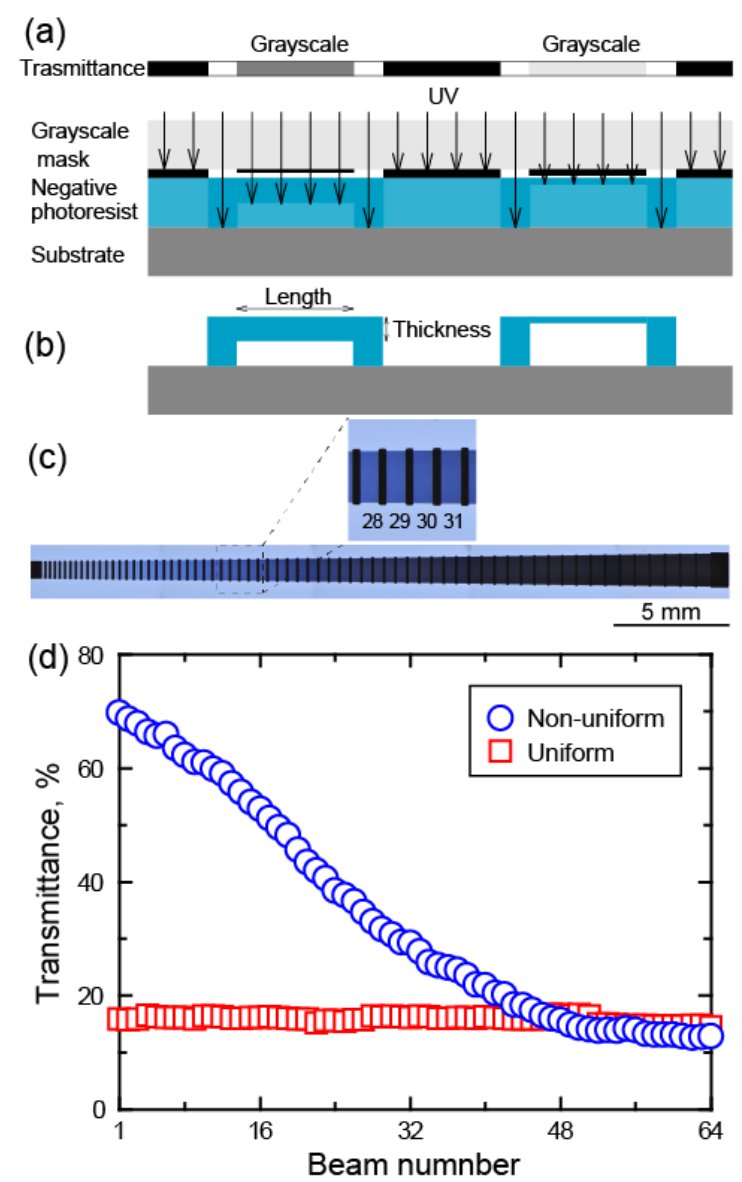

Figure 1: Schematic of the steps involved in the fabrication process: (a) during exposure and (b) after the development. (c) Photograph of a gray-scale mask and (d) Transmittance of the gray-scale patterns.

Figure 1 (c) shows a photograph of the photomask taken with transillumination. The length and the width of beam were changed from 750 to $1500 \mu \mathrm{m}$ and from 100 to $600 \mu \mathrm{m}$, respectively. The black patterns and gray patterns, which are located alternately, were designed for open ends and beams, respectively. For this study, 64 beams were integrated in an area of $1.5 \mathrm{~mm} \times 30 \mathrm{~mm}$. The transmittance of the gray-scale patterns was spatially controlled using an Al thin film of non-uniform thickness, the fabrication of which is described in detail in the Appendix A. For convenience, we numbered the beams from left to right as No.1 No.64. Figure 1 (d) shows the transmittance of light through the gray-scale patterns for each beam. It could be observed that the transmittance monotonically decreases with increase in beam number. 
In addition, we also prepared a photomask with uniform transmittance (Fig. 1(d)) to fabricate an MBA with uniform thickness. This was performed to investigate the effect of the thickness. The proposed MBA was fabricated using a negative photoresist, SU-8 3050 (MicroChem). In the typical process, an SU-8 film of thickness $200 \mu \mathrm{m}$ was spin-coated onto a silicon substrate under the following conditions: $500 \mathrm{rpm}$ for $5 \mathrm{~s}$, ramp up for $4 \mathrm{~s}$, and 800 rpm for 30 s. Subsequently, the spin-coated SU-8 film was soft baked at $95{ }^{\circ} \mathrm{C}$ for $60 \mathrm{~min}$. Following that, the SU-8 film was exposed to modified UV light through the gray-scale mask with a $150 \mathrm{~kJ} / \mathrm{m}^{2}$ dose. After exposure, the film was baked at $65{ }^{\circ} \mathrm{C}$ for $1 \mathrm{~min}$ and at $95{ }^{\circ} \mathrm{C}$ for 10 min. Finally SU-8 was developed in a developer (MicroChem) for 50 min under constant agitation of the developer using a magnetic stirrer. After the development, we rinse the MBA with isopropyl alcohol. 


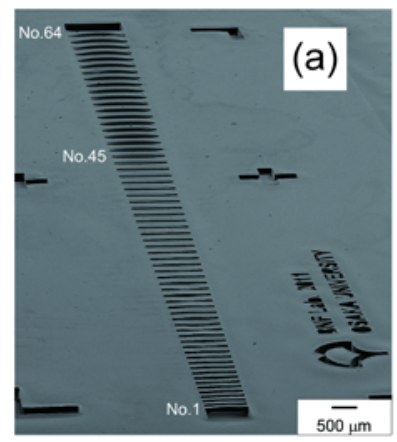

(b)

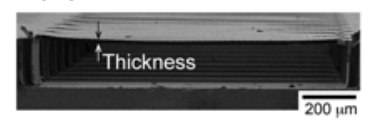

(c)
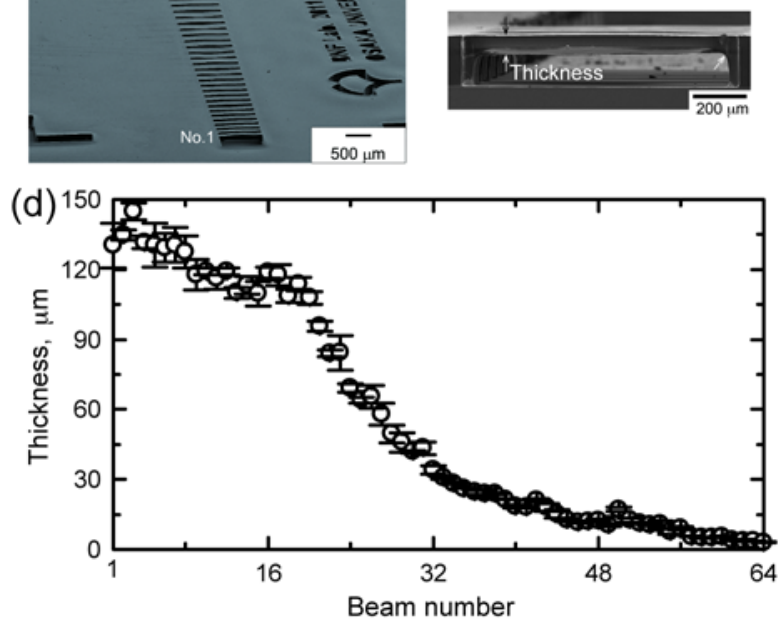

Figure 2: (a) Scanning electron microscopic image of MBA with non-uniform thickness, Cross-sectional SEM image of (b) Beam No. 42 (c) Beam No. 25 (d) Plot showing the variation in the thickness of beams

\section{Results and Discussion}

Figure 2(a) shows the scanning electron microscopic (SEM) image of an MBA with non-uniform thickness. In this image, the beam number increases from the bottom to the top. The relatively thicker beams, numbered 1-44, seemed to exhibit less initial deformation, while thin beams, numbered 45-64, showed deformation. This deformation might have originated from the residual stress induced during the fabrication process.

Furthermore, the thickness was evaluated by breaking the microbeams, and observing the beams along the side by using SEM. Figures 2(b) and 2(c) show the enlarged view of the beam No. 42 and 25, respectively. As evidenced from the SEM image, the thickness of each beam was almost uniform in the axial direction. The thicker beam, No. 25, showed rounded corners at the boundary on the underside. However, the thinner beam, No.42, had sharper 
corner, as evidenced from Fig. 2(b). This implies that the contrast becomes lower with increase in the penetration depth of UV. Moreover, as shown in Fig. 2(d), the thickness of the beams changed from $142 \mu \mathrm{m}$ to $2.99 \mu \mathrm{m}$, through 64 beams. The error bar in the data shows the thickness variation in each beam in the axial direction. The relatively large error bars in thicker beams (thickness $>60 \mu \mathrm{m}$ ) could be attributed to the relatively low contrast in thicker beams.

In order to demonstrate the further performance of the fabrication process, we have optimized the process conditions for the wider thickness range 2.99-142 $\mu \mathrm{m}$ compared to the previous report of 6.6-49 $\mu \mathrm{m}^{11}$ by modifying the transmittance of the gray-scale mask. In addition, we have prolonged the soft bake time from $45 \mathrm{~min}$ to $60 \mathrm{~min}$ to prevent the grayscale mask from sticking to the SU-8 surface at the exposure. These modifications may contribute to change in the residual stress and the range of the resonant frequency. However, the detailed investigation on the effect of the process condition is under way and we hope to include it in our future report. 

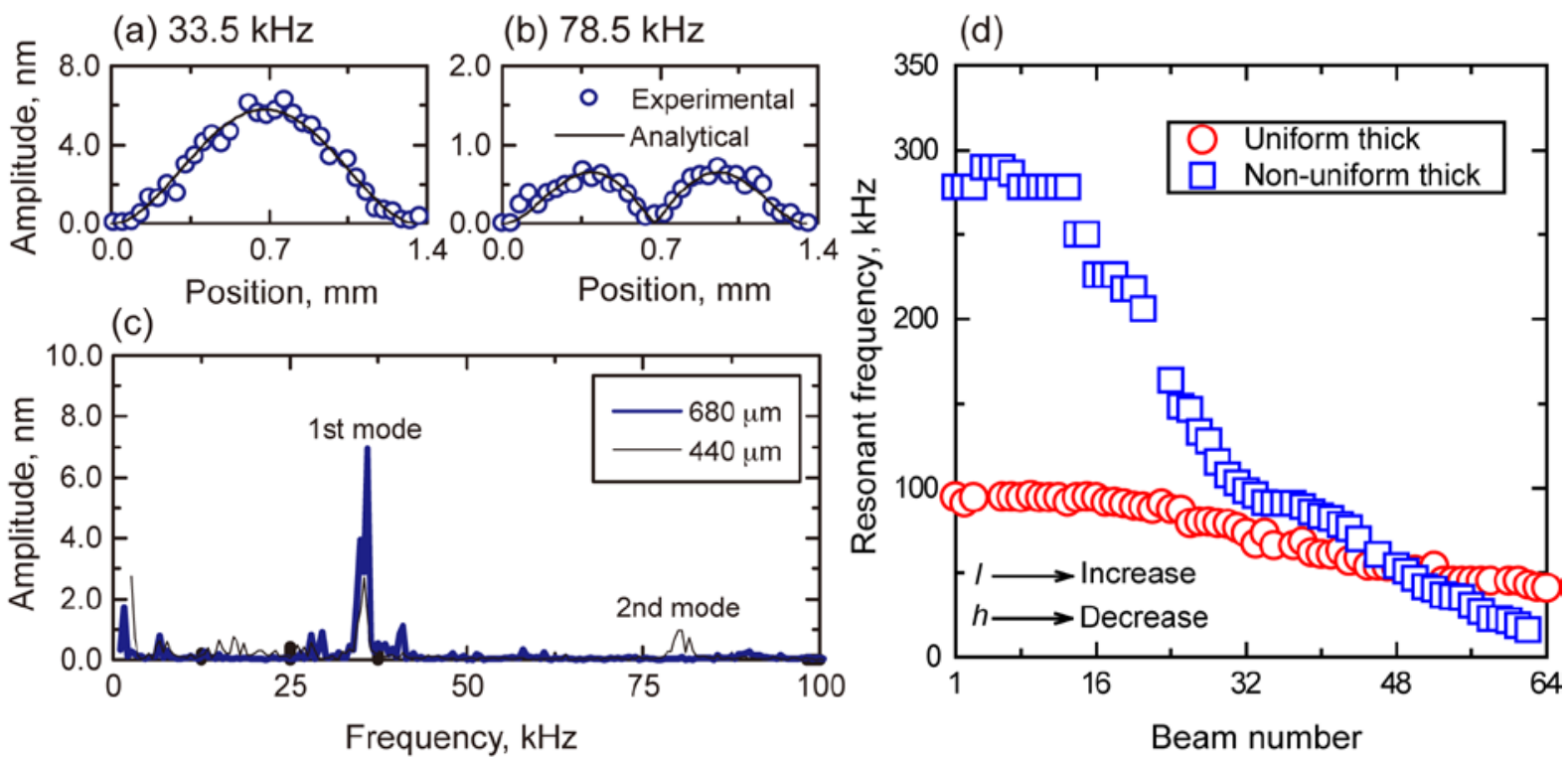

Figure 3 Spatial distribution of vibration amplitude of beam No. 55 at (a) $33.5 \mathrm{kHz}$ and (b) $78.5 \mathrm{kHz}$. (c) The frequency response of the vibration amplitude measured at $680 \mu \mathrm{m}$ and 440 $\mu m$. (d) Comparison of the resonant frequency of uniform and non-uniform thick MBAs.

Additionally, the vibration characteristics of microbeams were investigated by measuring the out-of-plane vibration of the beams using a laser Doppler vibrometer. In-depth details on the measuring system has been reported by us in our previous paper ${ }^{2}$. Briefly, we here applied a pure tone sound with a sound pressure of $80 \mathrm{dBL}$ to MBA and measured the vibration of the beam. The amplitude of the vibration was in the order of nm that was small compared to the geometry of the beams. We observed the sinusoidal vibration of beams at all the frequency we examined. The frequency spectrum of the vibration showed a clear single peak at the frequency of the applied sound. Figures 3(a) and 3(b) show the spatial distribution of vibration amplitude for beam No. 55 at $33.5 \mathrm{kHz}$ and $78.5 \mathrm{kHz}$, respectively, along the axial direction. Here $680 \mu \mathrm{m}$ is the center of the beam. The distributions qualitatively agreed well with the analytical solutions of the bending vibration of an Euler-Bernoulli beam with both ends fixed condition for the first mode and the second mode, respectively. This result 
implies that the vibration is dominated by the bending mode and the thickness change in the MBA contributes to widening of the frequency range.

Figure 3(c) shows the frequency responses of the beam No. 55 measured at $680 \mu \mathrm{m}$ and $440 \mu \mathrm{m}$. The data labeled as $680 \mu \mathrm{m}$ shows a clear peak at $33.5 \mathrm{kHz}$, which corresponds to the resonant frequency of the first mode. We did not observe significant nonlinear response of the beam such as harmonic distortion up to $120 \mathrm{dBL}$ that was the maximum sound pressure with the current experimental setup. Therefore, we confirmed the MBA is practical for the resonant sensing under the sound pressure level below 120 dBL. Following that, we focused on the first mode vibration and measured the frequency response of the vibration amplitude at the center of the microbeams. Figure 3 (d) shows the first mode resonant frequencies of each beam in the MBA. As evidenced from the figure, the resonant frequency is higher for small beam numbers and it decreases with increase in beam number.

In order to elucidate the reliability of the proposed technique, we fabricated several MBAs with the same design and evaluated the variations in the thickness and the resonant frequency among the different MBAs. The variations were $6.2 \%$ both in the thickness and the resonant frequency.

To understand the effect of thickness on the resonant frequency, the obtained result was compared with that of the MBA with a uniform thickness of $18 \mu \mathrm{m}$ (Fig. 4(d)). The comparison plot clearly shows the MBA with non-uniform thickness exhibits wider frequency range than that of the MBA of uniform thickness. The resonant frequency of the Euler-Bernoulli beam could be theoretically predicted as $f=\left(h \beta^{2} / 4 \pi l^{2}\right)(E / 3 \rho)^{0.5}$, where $h, l, E$, and $\rho$ correspond to the thickness, length, Young's modulus, and density of the beam, respectively. The value of $\beta$ is assumed to be 4.73 for the both end fixed boundary condition and the first mode vibration. The abovementioned relation indicates that the resonant frequency is inversely proportional to the square of the beam length and directly proportional 
to the beam thickness. Assuming that the value of $E$, $l$, and $\rho$ are the same between nonuniform and uniform MBAs, the effect of the thickness on the frequency range could be evaluated from the ratio $r_{\mathrm{f}}=f_{\max } / f_{\min }$, where $f_{\max }$ and $f_{\min }$ are the maximum and minimum frequency of each MBA, respectively. Ideally, the ratio $r_{\mathrm{f}}$ becomes $\left(l_{64} / l_{1}\right)^{2}$ and $\left(h_{1} / h_{64}\right)\left(l_{64} / l_{1}\right)^{2}$ for the MBA with uniform and non-uniform thickness, respectively. Here, the subscript indicates the beam number.

The experimental value of $r_{\mathrm{f}}$ was estimated to be 2.32 and 25.2 for the MBA with uniform and non-uniform thickness, respectively. This implies that the frequency range of the MBA gets widened by a factor of 10.9 with changes in the thickness of the beams. However, theoretical calculations based on the Euler-Bernoulli beam predicts that the thickness change should widen the frequency range by a factor of $\left(h_{1} / h_{64}\right)$, which is 44 . This is larger than the experimentally observed value. In addition, $r_{\mathrm{f}}$ for the MBA with uniform thickness should be $\left(l_{64} / l_{1}\right)^{2}$, which equal to a value of 4 .

The observed discrepancy between the theoretical prediction and the experimental result could be hypothesized as due to the tensile stress in microbeams. The effect of the residual stress was analyzed by fabricating another device of various thick cantilever beams that are free of tensile residual stress in axial direction. Also, in this device, we integrated both ends fixed beams with the same width, length, and thickness of the cantilevers. Following that, we measured the resonant frequency of cantilever beams and estimated the tensile residual stress in both ends fixed beams (See Appendix B for detailed method). The estimated tensile residual stress increased from 5.74 $\mathrm{MPa}$ to $17.0 \mathrm{MPa}$ within the thickness ranging from $13.0 \mu \mathrm{m}$ to $36.2 \mu \mathrm{m}$ (See Fig.S3). The resonant frequency was then predicted, taking into account the tensile residual stress using the Rayleigh method. The resonant frequency with the effect of the tensile residual stress can be written as 
$f=\frac{1}{2 \pi} \sqrt{\frac{\int_{0}^{l}\left[E I\left(\partial^{2} y / \partial x^{2}\right)^{2}+T b h(\partial y / \partial x)^{2}\right] d x}{\int_{0}^{l} \rho b h y^{2} d x}}$

where $I, T$, and $y$ are the moment of inertia of area, tensile residual stress, and deflection curve equation, respectively. The deflection curve equations for the both ends fixed beams were estimated using the following equations:

$y=\frac{y_{0}}{2}\left(1-\cos \frac{2 \pi}{l} x\right) \quad 0 \leq x \leq l$

where $y_{0}$ is the maximum vibration amplitude of the beams. The estimated resonant frequency with $E=5.4 \mathrm{GPa}$ and $\rho=1153 \mathrm{~kg} / \mathrm{m}^{3}$ was compared with the experimental data in

Fig. 4. The comparison shows that the accuracy of the prediction was greatly improved in thin beams, with beam number ranging from 32 to 64 for the MBA with non-uniform thickness. In case of the MBA with a uniform thickness of $18 \mu \mathrm{m}$, the predictions were in good agreement for all the beams. This result indicates that the discrepancy between the theoretically predicted and the experimentally observed results, occurring in case of thin beams, is caused by the tensile residual stress. This result leads to the conclusion that the tensile residual stress, especially in thin beams limits the frequency range of the MBA. On the other hand, the effect of the tensile residual stress was observed to be insignificant in thicker beams No. 1-20, where the prediction even without the effect of the residual stress overestimated in these beams. This overestimation is considered as the effect of shear stress that is neglected in the Euler-Bernoulli theory because in this range the thickness becomes larger and length becomes smaller.

The MBA was designed to work in a liquid to decrease and approximate the resonant frequencies to CF of human being. Therefore, the resonant frequency in air was relatively high. We expect the resonant frequency of beam No.64 in water becomes around $200 \mathrm{~Hz}$ with the effect of the added mass due to surrounding fluid ${ }^{2}$. In order to further decrease the resonant frequency to $20 \mathrm{~Hz}$, we need either to decrease the thickness one order of magnitude 
or to increase the length of the beam $10^{0.5}$ folds. However, fabricating such a thin or long beam is still challenging with the proposed technique. The main difficulty in the fabrication is due to the beam sticking that occurs when the MBA is removed from the isopropyl alcohol. We believe that we can overcome this by developing a beam releasing technique such as freeze-drying ${ }^{22}$. We also believe that the tensile residual stress could be reduced by optimizing the process conditions. Further research on the optimization of the processing conditions is under way, and we hope to include it in our future report.

In our previous study ${ }^{8,10}$, we developed a MEMS fabricated acoustic sensor with a poly(vinylidene fluoride-trifluoroethylene) (P(VDF-TrFE)) film. $\mathrm{P}(\mathrm{VDF}-\mathrm{TrFE})$ is a piezoelectric material that can be deposited with a spin-coating method. The cure temperature is below $145{ }^{\circ} \mathrm{C}$ that is lower than the glass temperature of the SU-8. In addition, we have confirmed the feasibility of deposition of metal thin films on the MBA with a thermal deposition. These facts imply that fabricating a P(VDF-TrFE) film on the MBA is possible and a piezoelectric acoustic sensor based on the MBA is promising. Here, we have proposed the MBA as an acoustic sensor. The fabrication process was fully based on the standard photolithography processes and it was compatible with standard microfabrication techniques. Therefore, we believe the MBA can be also used for the substrates of other applications such as a MEMS based energy harvesting ${ }^{23,24}$. 


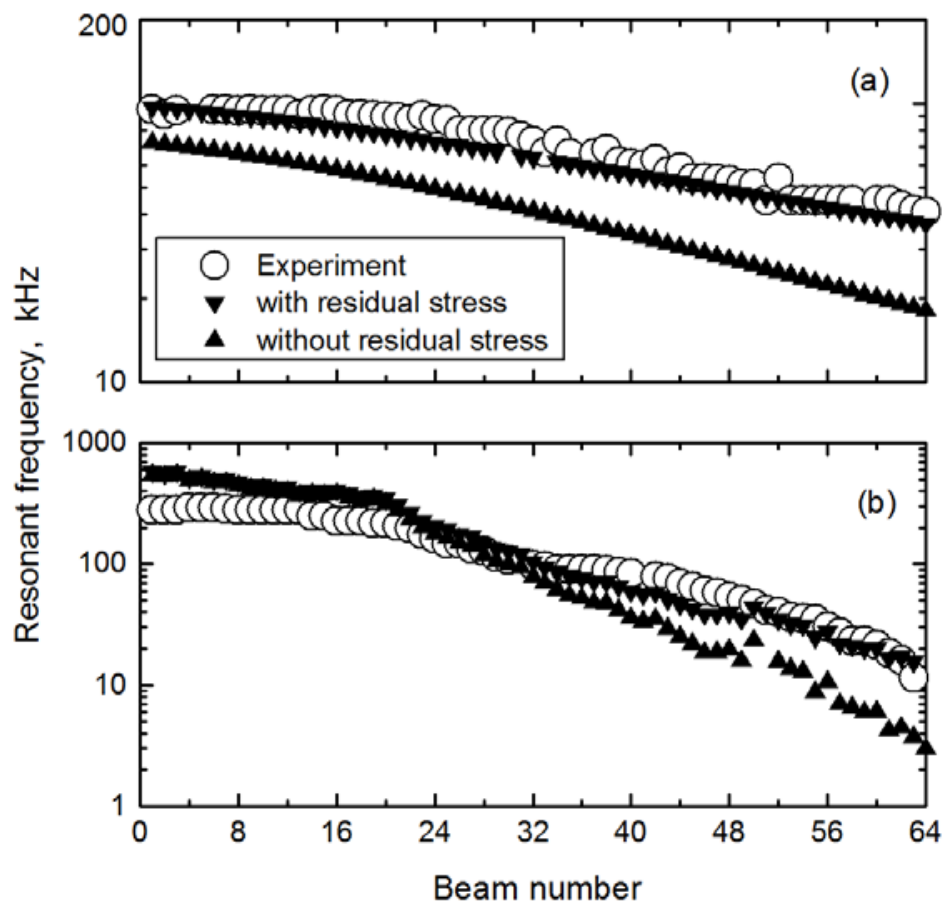

Figure 4 Comparison of resonant frequency for MBA with (a) uniform and (b) non-uniform thickness. Predictions were made with and without taking into account the tensile residual stress.

\section{Concluding Remarks}

In summary, we have fabricated an MBA with non-uniform thickness, and analyzed the effect of thickness on the frequency range of the MBA. The frequency range of the MBA with non-uniform thickness was found to be 10.9 times that of the MBA with uniform thickness. The effect of tensile residual stress was observed to be significant for thin beams and limits the frequency range of the MBA. This requires optimization of the processing conditions. 


\section{Acknowledgement}

This work was supported by a Grant-in-Aid for Research on Sensory and Communicative Disorders, funded by the Japanese Ministry of Health, Labor and Welfare. The authors would like to acknowledge the valuable contributions of Dr. Juichi Ito and Dr. Takayuki Nakagawa, Kyoto University, and Dr. Shinji Hamanishi, Sendai National College of Technology.

\section{Appendices}

\section{Appendix A. Fabrication of grayscale mask using non-uniform thick} metal film

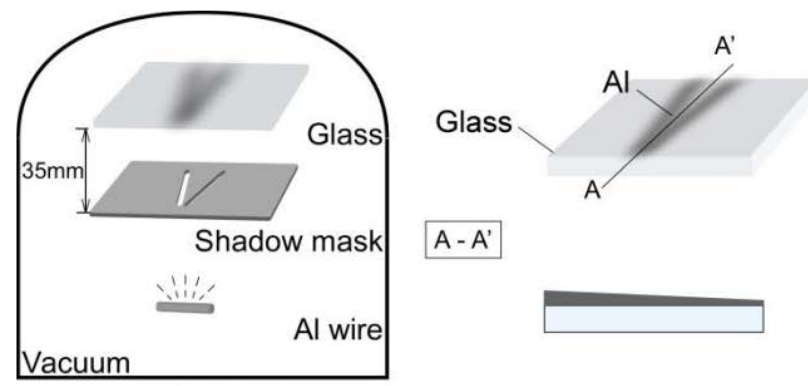

(a) Al deposition for gray region

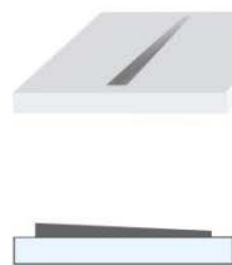

(c) Al etching

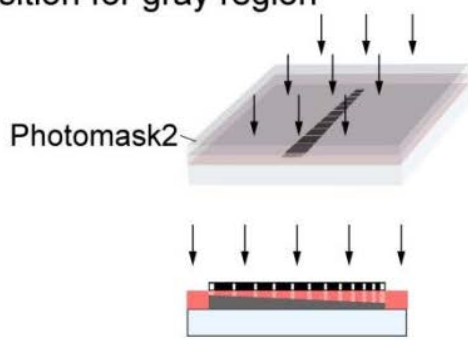

(d) Exposure \& Development

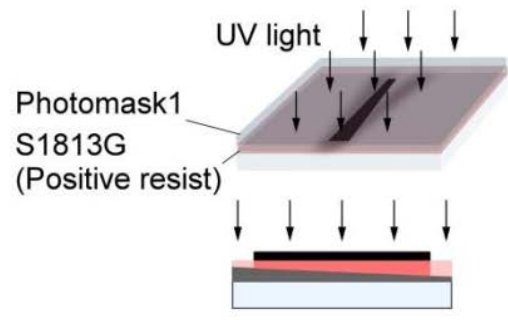

(b) Exposure \& Development

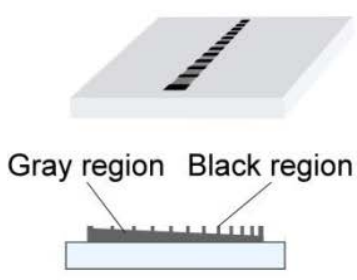

(e) Al deposition \& Lift off for black region

Figure S1: Schematic illustration of the steps involved in the fabrication of grayscale mask:(a) deposition of non-uniform thick Al thin film by thermal deposition, (b) patterning of gray regions by photolithography and (c) etching, (d) patterning of black regions in photomask by lift-off process.

The schematic of the steps involved in the fabrication of the grayscale mask is shown in Figure S1. In order to form a non-uniform thick metal film on a glass substrate, we placed a 
shadow mask between the substrate and the evaporation source, at a distance of $35 \mathrm{~mm}$ apart from the substrate. The shadow mask has V-like through-hole, though which the metal vapor can pass. On the substrate, the metal vapor forms V-shaped film, which was diffused due to the distance between the substrate and the shadow mask. Along the line AA' shown in Fig. S1(a), the diffused metal film forms an overlapped region that provides the non-uniform thick film for the fabrication of the grayscale mask. Subsequently, the metal film was patterned by photolithography using a positive photoresist (S1813G, Shipley), followed by etching, as shown in Fig.S1(b) and (c). Finally, the black patterns made of thick metal films were formed by a lift-off process, as shown in Fig. S1(d) and (e).

\section{Appendix B. Measurement of tensile residual stress in both ends fixed beams}

As a part of our study, we experimentally quantified the tensile residual stress by comparing the resonant frequencies of cantilever and both ends fixed beams. The deflection curve equations for cantilever were estimated using the following equation:

$y=y_{0} \frac{x^{2}}{l^{2}} \quad 0 \leq x \leq l$

Assuming $T=0$ in Eq.(1) in case of cantilever beams, we obtained the sound velocity $\sqrt{E / \rho}$, as shown in Fig. S2. The experimentally obtained sound velocity was found to be in good agreement with the estimated values with $E=5.4 \mathrm{GPa}^{25}$ and $\rho=1153 \mathrm{~kg} / \mathrm{m}^{3}$, implying the validity of the measurement. Using the sound velocity, we further estimated the tensile residual stress in both ends fixed beams as shown in Fig. S3. The data thus obtained shows that the tensile residual stress increases from 5.74 MPa to 17.0 MPa, with the thickness in the range from $13.0 \mu \mathrm{m}$ to $36.2 \mu \mathrm{m}$. Quantitatively, this observation exhibited the trend similar to that reported in a literature ${ }^{26}$. Since we could measure the tensile residual stress in the beams 
with abovementioned thicknesses, we extrapolated it by fitting a function $T=T_{0}$ (1-exp($\left.h / h_{0}\right)$ ), where $h_{0}$ and $T_{0}$ are experimentally determined as $150 \mu \mathrm{m}$ and $72 \mathrm{MPa}$, respectively.

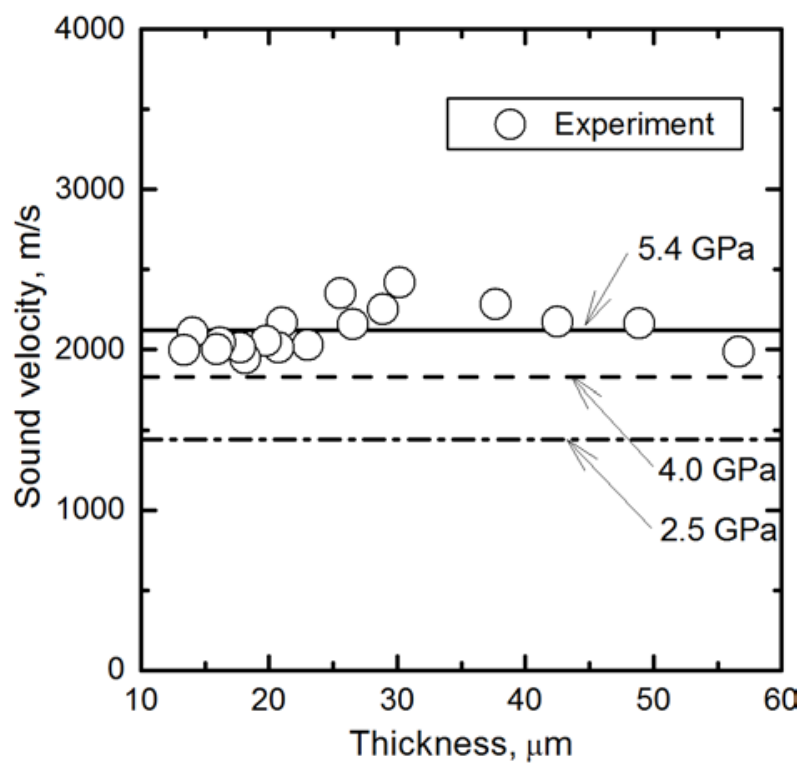

Figure S2: Sound velocity estimated from the resontant frequency of the cantilever beams.

Solid, broken, and dashed-dotted lines denote estimated sound velocity using $E=5.4 \mathrm{GPa}^{25}$, 4.0 $\mathrm{GPa}^{27}$, and $2.5 \mathrm{GPa}^{28}$, respectively.

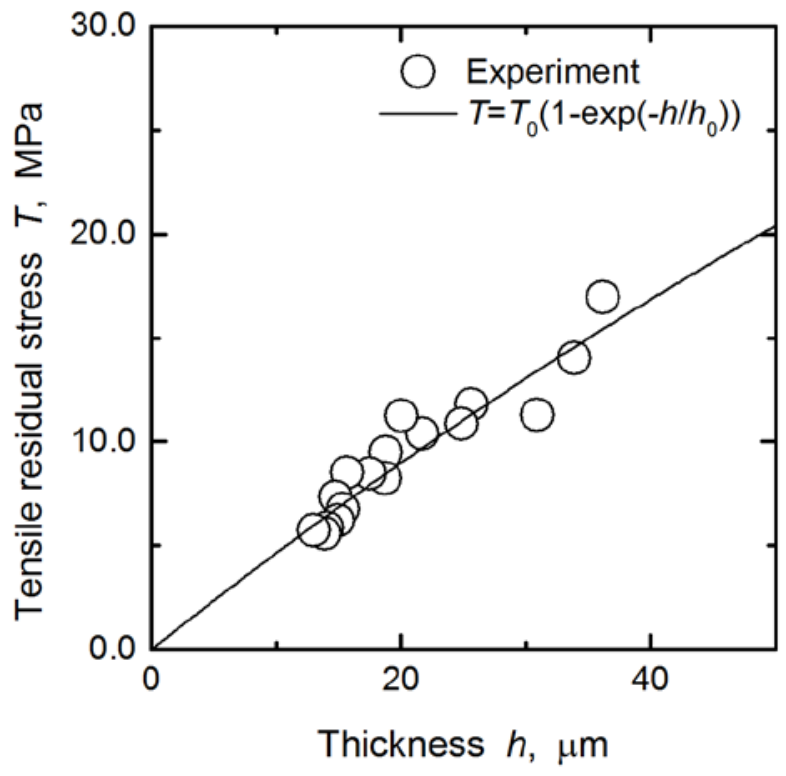

Figure S3: Tensile residual stress in both ends fixed beams plotted as a function of thickness 


\section{Reference}

1. F. Chen, H. I. Cohen, T. G. Bifano, J. Castle, J. Fortin, C. Kapusta, D. C. Mountain, A. Zosuls and A. E. Hubbard, J. Acoust. Soc. Am. 119 (1), 394-405 (2006).

2. H. Shintaku, T. Nakagawa, D. Kitagawa, H. Tanujaya, S. Kawano and I. Juichi, Sens. Act. A: Physical 158 (2), 183-192 (2010).

3. $\quad$ K. Tanaka, M. Abe and S. Ando, IEEE ASME Trans. Mechatron. 3 (2), 98-105 (1998).

4. $\quad$ R. D. White and K. Grosh, Proc. Natl. Acad. Sci. U.S.A. 102 (5), 1296-1301 (2005).

5. $\quad$ M. J. Wittbrodt, C. R. Steele and S. Puria, Audiol. Neurootol. 11 (2), 104-112 (2006).

6. T. Xu, M. Bachman, F. G. Zeng and G. P. Li, Sens. Act. A: Physical 114 (2-3), 176-182 (2004).

7. G. Zhou, L. Bintz, D. Z. Anderson and K. E. Bright, J. Acoust. Soc. Am. 93 (3), 1516-1523 (1993).

8. H. Shintaku, T. Tateno, N. Tsuchioka, H. Tanujaya, J. Ito and S. Kawano, J. Biomech. Sci. Eng. 5 (3), 229-235 (2010).

9. H. Shintaku, T. Inaoka, T. Nakagawa, S. Kawano and J. Ito, Journal of Biomechanical Science and Engineering 8 (3), 198-208 (2013).

10. T. Inaoka, H. Shintaku, T. Nakagawa, S. Kawano, H. Ogita, T. Sakamoto, S. Hamanishi, H. Wada and J. Ito, Proc. Natl. Acad. Sci. U.S.A. 108 (45), 18390-18395 (2011).

11. H. Shintaku and S. Kawano, Proceedings of ASME-JSME-KSME Joint Fluids Engineering Conference, (36038-36031)-(36038-36032) (2011).

12. T. Kobayashi, K. Zusho, H. Shintaku and S. Kawano, Proceedings of 2012 ASME-ISPS /JSME-IIP Joint International Conference on Micromechatronics for Information and Precision Equipment MIPE2012, 285-287 (2012).

13. J. Chung and W. Hsu, J.of Vac. Sci. Technol. B: Microelectro. and Nano. Struct. 25 (5), 1671-1678 (2007).

14. J. A. Lee, S. W. Lee, K.-C. Lee, S. I. Park and S. S. Lee, J. Micromech. Microeng. 18 (3), 035012 (2008).

15. A. Mata, A. J. Fleischman and S. Roy, J. Micromech. Microeng. 16 (2), 276 (2006).

16. H. Yu, B. Li and X. Zhang, Sens. Act. A: Physical 125 (2), 553 - 564 (2006).

17. M. Han, W. Lee, S.-K. Lee and S. S. Lee, Sens. Act. A: Physical 111 (1), 14 - 20 (2004).

18. J. M. Dykes, D. K. Poon, J. Wang, D. Sameoto, J. T. K. Tsui, C. C. amd G.H. Chapman, A. M. Parameswaren and B. L. Gray, Proc. of SPIE 6465 (-), (64650N-64651-64650N-64612) (2007).

19. K. Totsu, K. Fujishiro, S. Tanaka and M. Esashi, Sens. Act. A: Physical 130 (-), 387 - 392 (2006).

20. C. M. Waits, A. Modafe and R. Ghodssi, J. Micromech. Microeng. 13 (2), 170 (2003).

21. Y. Hirai, Y. Inamoto, K. Sugano, T. Tsuchiya and O. Tabata, J. Micromech. Microeng. 17 (2), 199 (2007).

22. H. Guckel, J. J. Sniegowski, T. R. Christenson, S. Mohney and T. F. Kelly, Sensor Actuator 20 (1-2), 117-122 (1989).

23. K. Morimoto, I. Kanno, K. Wasa and H. Kotera, Sensor Actuat a-Phys 163 (1), 428-432 (2010).

24. K. Wasa, T. Matsushima, H. Adachi, I. Kanno and H. Kotera, J Microelectromech S 21 (2), 451-457 (2012).

25. H. Yu, U. Balogun, B. Li, T. W. Murray and X. Zhang, J Micromech Microeng 14 (11), 1576-1584 (2004).

26. R. L. Barber, M. K. Ghantasala, R. Divan, K. D. Vora, E. C. Harvey and D. C. Mancini, Microsyst Technol 11 (4-5), 303-310 (2005).

27. A. del Campo and C. Greiner, J Micromech Microeng 17 (6), R81-R95 (2007).

28. M. Hopcroft, T. Kramer, G. Kim, K. Takashima, Y. Higo, D. Moore and J. Brugger, Fatigue Fract Eng M 28 (8), 735-742 (2005). 